\title{
Pattern Formation in Active Fluids
}

\author{
Justin S. Bois, ${ }^{1,2}$ Frank Jülicher, ${ }^{1}$ and Stephan W. Grill ${ }^{1,2}$ \\ ${ }^{1}$ Max Planck Institute for the Physics of Complex Systems, Nöthnitzer Straße 38, D-01187 Dresden, Germany \\ ${ }^{2}$ Max Planck Institute of Molecular Cell Biology and Genetics, Pfotenhauerstraße 108, D-01307 Dresden, Germany
}

(Received 14 October 2010; published 13 January 2011)

\begin{abstract}
We discuss pattern formation in active fluids in which active stress is regulated by diffusing molecular components. Nonhomogeneous active stress profiles create patterns of flow which transport stress regulators by advection. Our work is motivated by the dynamics of the actomyosin cell cortex in which biochemical pathways regulate active stress. We present a mechanism in which a single diffusing species up regulates active stress, resulting in steady flow and concentration patterns. We also discuss general pattern-formation behaviors of reaction-diffusion systems placed in active fluids.
\end{abstract}

DOI: 10.1103/PhysRevLett.106.028103

In his seminal 1952 paper, Turing proposed that reactiondiffusion mechanisms are responsible for pattern formation in developing organisms [1]. He remarked that mechanical stresses affect chemical patterning and should also be considered. In a biological context, such stresses can, for example, be generated by motor proteins in the cell cytoskeleton, driven by hydrolysis of adenosine triphosphate. The dynamic reorganization of the cytoskeleton far from thermal equilibrium has been identified as an integral part of many aspects of cell and tissue patterning [2].

It has been argued that the cytoskeleton is an example of an active fluid [3]. Here, we discuss general phenomena of pattern formation in active fluids that involves actively generated hydrodynamic flows which advect chemical species that control active stresses and may undergo chemical reactions. We develop a general framework describing such processes, based on hydrodynamic descriptions of active fluids or gels [3-6]. As a simple example, we consider pattern formation in a thin film of active fluid of constant thickness $h$ where movements occur only along the $x$ axis. This one-dimensional scenario is motivated by the cell cortex, a thin layer of active gel associated with the cell membrane which exhibits flows and maintains constant thickness through exchange of material with the surrounding cytoplasm [6,7]. Here, we ignore viscoelasticity and treat the material as isotropic. A key ingredient is that the active stress is a function of the concentrations of regulatory chemical species that obey reaction-diffusionadvection equations.

We first consider the case where the fluid contains a single regulator of active stress with concentration $c(x, t)$. The concentration of the regulator is governed by a onedimensional conservation law [8]:

$$
\partial_{t} c=-\partial_{x} j, \quad j=-D \partial_{x} c+v c,
$$

where the flux $j$ of the regulator has a diffusive component with diffusion coefficient $D$ and an advective component with bulk flow velocity $v$. The constitutive equation of the active fluid is $[6,7]$
PACS numbers: 87.16.Uv, 87.10.Ca, 87.16.Ln, 89.75.Kd

$$
\sigma=\eta \partial_{x} v+\zeta \Delta \mu
$$

The total stress $\sigma$ consists of viscous stress $\eta \partial_{x} v$, where $\eta$ is the viscosity, and $c$-dependent active stress $\zeta \Delta \mu(c)$, positive for contraction and dependent on the change in chemical potential associated with adenosine triphosphate hydrolysis, $\Delta \mu$. Neglecting inertial forces, typically valid on a cellular scale, we write the force balance as

$$
\partial_{x} \sigma=\gamma v
$$

where we have introduced a friction coefficient $\gamma$ to account for relative motion against a cell membrane or substrate. Taken together, (1)-(3) state that advective transport and concentration dependence of the active stress couple the spatial distribution of the regulator to active fluid motion.

In characterizing the system, we restrict ourselves to finite domains with $x \in[0, L]$ with periodic or no-flux boundary conditions. For both types of boundary conditions, the total amount of regulator is conserved and the average concentration

$$
c_{0}=\frac{1}{L} \int_{0}^{L} d x c(x, t)
$$

is constant. Therefore, $\left[c(x)=c_{0}, v=0\right]$ is the unique homogeneous steady state.

To determine the nonhomogeneous steady states, we seek solutions of (1)-(3) with $\partial_{t} c=0$. Assuming a constant diffusion coefficient, we may integrate (1) to solve for the flow velocity, giving

$$
v=D \partial_{x} \ln c+j_{0} / c,
$$

where $j_{0}$ is a constant of integration representing a constant flux through the system. For no-flux boundary conditions, $v(0)=\partial_{x} c(0)=0$ requires that $j_{0}=0$. For periodic boundary conditions, it is instructive to use (5) in (3) and integrate with respect to $x$. Assuming constant $\gamma$, we obtain 


$$
\sigma=\sigma_{0}+\gamma D \ln c+\gamma j_{0} \int_{0}^{x} \frac{d x^{\prime}}{c\left(x^{\prime}\right)}
$$

where $\sigma_{0}$ is a constant of integration. Because

$$
j_{0} \int_{0}^{L} \frac{d x}{c(x)}=\frac{\sigma(L)-\sigma(0)}{\gamma}-D \ln \frac{c(L)}{c(0)},
$$

periodic boundary conditions require $j_{0}=0$. Thus, the steady state velocity for both no-flux and periodic boundary conditions is

$$
v=D \partial_{x} \ln c=D \partial_{x} u,
$$

where we have defined $u \equiv \ln c$ for notational convenience.

We use (6) and (8) in (2) to obtain an ordinary differential equation describing steady concentration profiles:

$$
\partial_{x}^{2} u=\ell^{-2} u+(D \eta)^{-1}\left[\sigma_{0}-\zeta \Delta \mu(u)\right] .
$$

Here, $\ell \equiv \sqrt{\eta / \gamma}$ is the characteristic length over which fluid velocity decays from a local active stress gradient [7]. The right-hand side may be written as $-\partial_{u} V(u)$, revealing that for constant parameters (9) has the form of a conservative anharmonic oscillator in which $x$ is the "time variable" and $u$ is the "space variable" with Hamiltonian

$$
\begin{aligned}
H\left(u, \partial_{x} u\right) & =\frac{1}{2}\left(\partial_{x} u\right)^{2}+V(u), \\
V(u) & =-\frac{u^{2}}{2 \ell^{2}}-\frac{1}{D \eta}\left(\sigma_{0} u-\int_{0}^{u} d u^{\prime} \zeta \Delta \mu\left(u^{\prime}\right)\right) .
\end{aligned}
$$

This analogy is instructive in investigating the nature of the steady states because we can employ known properties of conservative Hamiltonian systems [9]. In particular, nonhomogeneous steady states featuring a patterned regulator correspond to "oscillations" of $u(x)$ with $u$ varying between two roots, $u_{1}$ and $u_{2}$, of $E-V(u)=0$, where $E$ is the conserved value of $H$ along $u(x)$. For oscillations, the roots satisfy $u_{1}<\ln c_{0}<u_{2}$. The trajectory of the oscillations must satisfy (4) and have period $2 L / N$ with $N / \beta \in$ $\mathbb{Z}_{>0}$, where $\beta=1$ for no-flux and $\beta=2$ for periodic boundary conditions. The resulting regulator concentration profile consists of $N+1$ evenly spaced symmetric extrema with peaks of concentration $c_{\max }=e^{u_{2}}$ separated by valleys of concentration $c_{\min }=e^{u_{1}}$. An example is depicted in Fig. 1, where we have chosen $\zeta \Delta \mu(c)=(\zeta \Delta \mu)_{0} f(c)$, where $(\zeta \Delta \mu)_{0}$ is a positive characteristic active stress and $f(c)=c /(1+c)$. The steady velocity profile, given by (8), crosses zero at the extrema of the concentration profile such that material flows into the peaks and out of the valleys (Fig. 1). The pattern is maintained by a balance of advective flux into the peaks and diffusive flux out of them, thereby creating a concentration pattern together with a steady state flow profile.

If $V(u)$ is strictly concave, i.e., $\partial_{u}^{2} V<0$ for all $u$, where $\partial_{u}^{2} V=\ell^{-2}\left(\operatorname{Pe}_{u} f-1\right)$, with $\operatorname{Pe} \equiv(\zeta \Delta \mu)_{0} / D \gamma$, then $u_{1}=u_{2}=\ln c_{0}$, allowing only the homogeneous concentration profile. Here, we have defined the Péclet number,
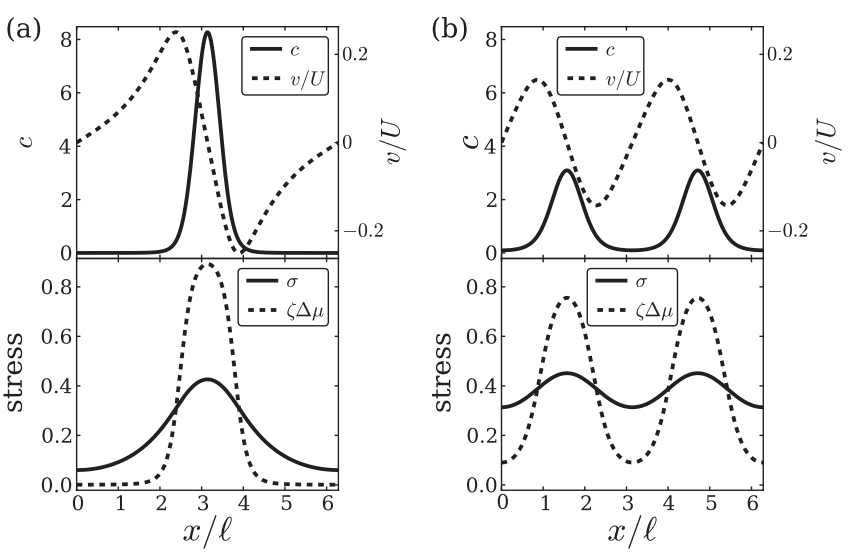

FIG. 1. The nonhomogeneous steady states with (a) one maximum and (b) two maxima for $\zeta \Delta \mu(c)=(\zeta \Delta \mu)_{0} c /(1+c)$, $\mathrm{Pe}=25, L / \ell=2 \pi$, and $c_{0}=1$, with periodic boundary conditions. The total stress $\sigma$ and active stress $\zeta \Delta \mu$ are given in units of $(\zeta \Delta \mu)_{0}$.

the ratio of diffusive to advective time scales: $\mathrm{Pe}=U \ell / D$, where $U=(\zeta \Delta \mu)_{0} / \sqrt{\eta \gamma}$ is the characteristic velocity, as evident from (2) and (3). If, however, $\partial_{c} f\left(c_{0}\right)>0$ (i.e., active stress is $u p$ regulated by the regulator) and $\mathrm{Pe}$ is sufficiently large, $V(u)$ is locally convex $\left(\partial_{u}^{2} V>0\right)$ at $\ln c_{0}$ and may allow oscillations that give nonhomogeneous steady states satisfying (4) with period $2 L / N$, as depicted in Fig. 2. The physical implication is that the active stress must be strong enough to promote advective flux into regions of high concentrations to counteract frictional resistance and diffusive counterflux.

To investigate the spontaneous emergence of flowaccompanied patterns from a quiescent homogeneous steady state, we perform linear stability analysis on the $c=c_{0}$ steady state. We consider a small perturbation of $\delta c=\delta c_{0} e^{\lambda(k) t+i k x}$, where $k= \pm \beta n \pi / L(n \in \mathbb{Z})$ is the wave number of the spatial perturbation. Inserting
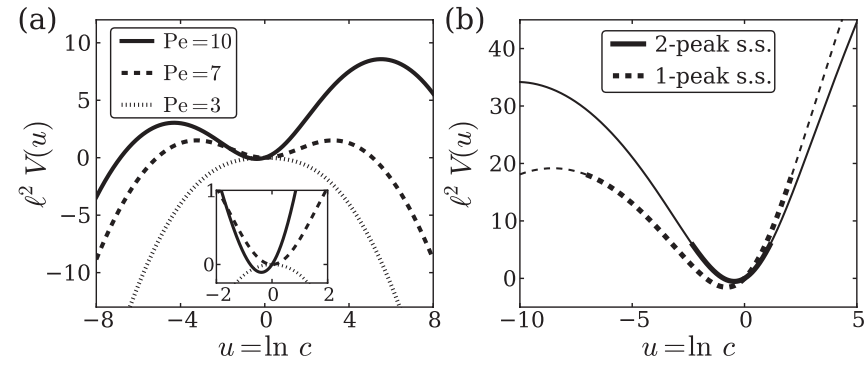

FIG. 2. (a) "Potentials" $V(u)$ for stable steady states with $\zeta \Delta \mu=(\zeta \Delta \mu)_{0} c /(1+c)$ and $c_{0}=1$. For small $\mathrm{Pe}, V(u)$ is concave; for moderate $\mathrm{Pe}, V(u)$ has a shallow minimum at $\ln c_{0}$; and for large Pe, $V(u)$ has a minimum at a $u<\ln c_{0}$ that is deep enough to support a nonhomogeneous steady state. Inset: Detail of the central extremum of $V(u)$. (b) Detail of relevant portions of potentials corresponding to the steady states depicted in Fig. $1(\mathrm{Pe}=25)$. The thick lines depict the portions of the potentials explored by the steady state. 
$c=c_{0}+\delta c$ into (2) and (3) and solving for the velocity gives, to linear order in $\delta c$,

$$
v(x)=\delta c \frac{i k(\zeta \Delta \mu)_{0}}{\gamma+\eta k^{2}} \partial_{c} f\left(c_{0}\right) .
$$

Inserting (11) into (1) and considering terms up to linear order in $\delta c$ reveals that the (real) eigenvalue, $\lambda(k)$, of the linearized system is given by the dispersion relation

$$
\lambda(k)=-k^{2} D\left(1-\frac{\operatorname{Pe} c_{0} \partial_{c} f\left(c_{0}\right)}{1+k^{2} \ell^{2}}\right) .
$$

As Pe grows, i.e., as the dynamics are more dominated by advection, instabilities of higher modes may grow, as shown in Fig. 3(a). The eigenvalue $\lambda(k)$ is positive for some $k$, signifying spontaneous formation of a nonhomogeneous concentration profile accompanied by flow, when

$$
\frac{\operatorname{Pe} c_{0} \partial_{c} f\left(c_{0}\right)}{1+(\beta \pi \ell / L)^{2}}>1
$$

A representative stability diagram is displayed in Fig. 3(b). Above a critical value of Pe, which is dependent on the average concentration $c_{0}$, the homogeneous state is unstable and patterns corresponding to the wave number $k_{\max }$ for which $\lambda(k)$ is maximal grow. Numerical integration of (1)-(3) demonstrates that, though the dynamics can initially approach a multipeaked steady state, such steady states are unstable and the system relaxes to the steady profile with a single peak, as shown in the example in Fig. 4. Depending on the parameters, this relaxation may occur over very long time scales, resulting in long-lived multipeaked profiles.

The simple scenario described above can be easily generalized for multiple diffusing species undergoing chemical reactions. Equation (1), augmented to include chemical reactions, holds for each species $i$ :

$$
j_{i}=-D_{i} \partial_{x} c_{i}+v c_{i}, \quad \partial_{t} c_{i}=-\partial_{x} j_{i}+R_{i} .
$$

Here, $R_{i}$ is the rate of production of species $i$ by chemical reaction and can depend on the concentration of any of the
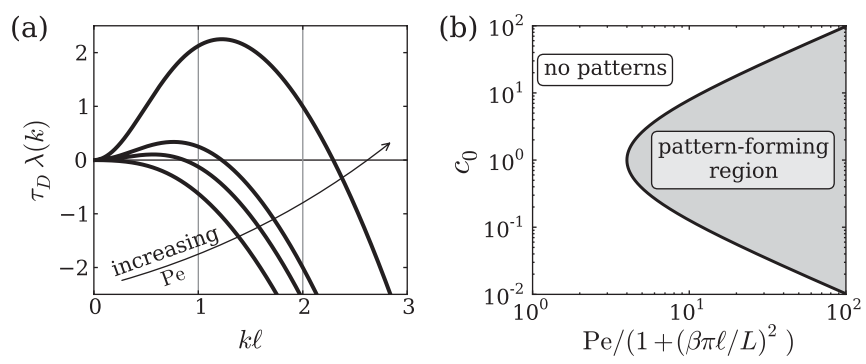

FIG. 3. (a) Dispersion relation for $\zeta \Delta \mu=(\zeta \Delta \mu)_{0} c /(1+c)$ and $c_{0}=1$ for $\mathrm{Pe}=3,7,10$, and 25, corresponding to plots depicted in Figs. 1, 2, and 4. The eigenvalue $\lambda(k)$ is nondimensionalized by the diffusive time scale $\tau_{D}=\ell^{2} / D$. For $L / \ell=2 \pi$ with periodic boundary conditions, as in Fig. $1, k \ell$ may take only integer values (vertical gray lines). (b) Linear stability diagram for the same active stress function. chemical species. The active stress may also depend on the concentrations: $\zeta \Delta \mu=(\zeta \Delta \mu)_{0} f\left(c_{1}, c_{2}, \ldots\right)$. We perform linear stability analysis on a homogeneous steady state, $\mathbf{c}_{0} \equiv\left(c_{1,0}, c_{2,0}, \ldots\right)$, in which the concentrations of all species are constant in space and the fluid is quiescent. The stability matrix $A$ has entries

$$
A_{i j}=-k^{2} D_{i} \delta_{i j}+\partial_{c_{j}} R_{i}\left(\mathbf{c}_{0}\right)+D_{1} \frac{k^{2} \mathrm{Pe} c_{i, 0} \partial_{c_{j}} f\left(\mathbf{c}_{0}\right)}{1+k^{2} \ell^{2}},
$$

where Pe is defined in terms of the diffusion coefficient of species 1 as $\mathrm{Pe}=(\zeta \Delta \mu)_{0} / D_{1} \gamma$. The homogeneous steady state is unstable, and the system can spontaneously form patterns in the chemical species accompanied by flow of the active fluid if $\operatorname{Re}[\lambda(k)]>0$ for some nonzero $k$, where $\lambda(k)$ is the eigenvalue of $\mathbf{A}$ with the largest real part. The first two terms in (15) comprise the standard expression for reaction-diffusion systems [10]. The last term is due to active stress-driven flow and notably depends on the wave number $k$, as opposed to reaction-diffusion systems in which only diffusive terms have $k$ dependence.

As an illustration, we consider the activator-substrate depletion model (ASDM) [11,12], a classic model which exhibits a Turing instability. The ASDM consists of an activator (with concentration $a$ ) and a substrate (with concentration $s$ ) that undergo chemical reactions with the following properties: (i) The activator is autocatalytic, consuming substrate in the process; (ii) the activator has a constant degradation rate; (iii) the substrate has a constant production rate. The simplest form of the chemical kinetics is $R_{a}=\rho_{a}\left(a^{2} s-a\right)$ and $R_{s}=\rho_{s}\left(1-a^{2} s\right)$, where $\rho_{a}$ and $\rho_{s}$ are chemical rate constants. The unique homogeneous steady state is $a_{0}=s_{0}=1$. When $\mathrm{Pe}=0$, or in the absence of active stress, we recover the classic ASDM, whose linear stability diagram is depicted in Fig. 5(a). In region I of parameter space, the substrate acts as a fast-diffusing "inhibitor" (since its depletion slows the production of the activator), the hallmark of a Turing instability. In the presence of advection due to active stress up regulation by the activator, the region of parameter space in which patterns form grows, as depicted

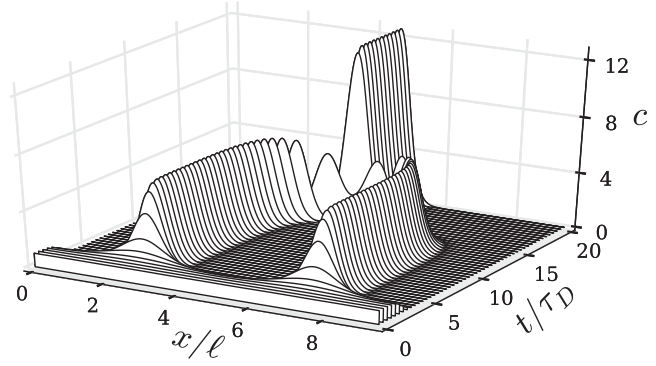

FIG. 4. Illustration of dynamics for the same system as in Fig. 1, except with $L / \ell=3 \pi$, such that the second mode grows fastest from the unstable homogeneous steady state. Time is nondimensionalized by $\tau_{D}=\ell^{2} / D$. 

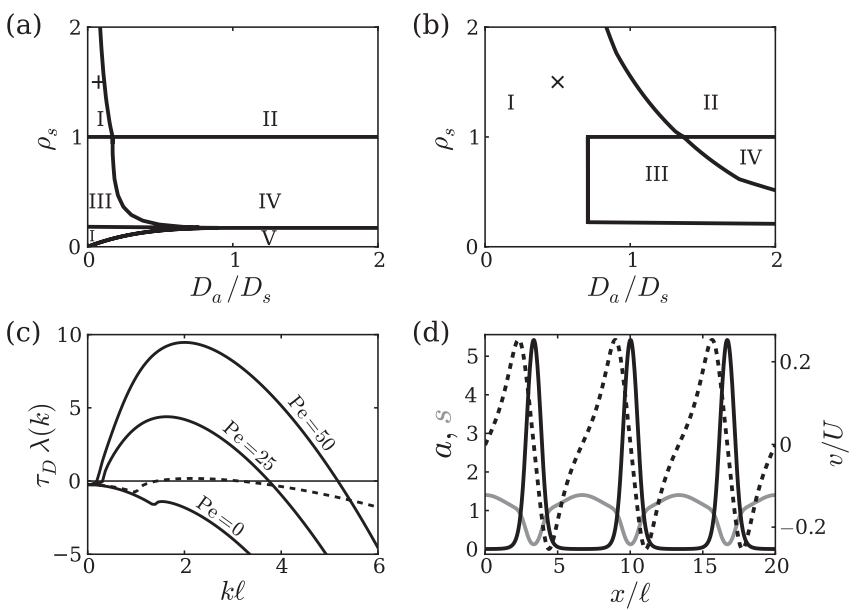

FIG. 5. Characterization of the ASDM. (a) Linear stability diagram in the absence of flow $(\mathrm{Pe}=0)$ for $\rho_{a}=1$, assuming $L / \ell \gg 1$. Region I: $\lambda(k)$ is real and positive with $\left|k_{\max }\right|>0$. This is the pattern-forming region. Region II: $\operatorname{Re}[\lambda(k)]<0$ (stable homogeneous steady state). Region III: $\lambda(k)$ is real and positive with maxima at $k=0$ and some $k \neq 0$. Patterns may form in this region, depending on initial conditions. Region IV: $\operatorname{Re}[\lambda(k)]>0, \quad \operatorname{Im}[\lambda(k)] \neq 0, \quad k_{\max }=0 \quad$ (oscillatory regime). Region V: $\lambda(k)$ real and positive with $k_{\max }=0$. (b) Linear stability diagram for $\mathrm{Pe}=7, \rho_{a}=1$, and $\zeta \Delta \mu /(\zeta \Delta \mu)_{0}=$ $a /(1+a)$. (c) Dispersion relation for the ASDM with $\lambda(k)$ nondimensionalized by $\tau_{D}=\ell^{2} / D_{s}$. Solid lines are for parameters given by the " $X$ " in (b). For reference, the dashed line is for $\mathrm{Pe}=0$ and parameters given by the " + " in (a). (d) Nonhomogeneous steady state for $\mathrm{Pe}=7$ and parameters given by the " $X$ " in (b) with periodic boundary conditions. The activator and substrate concentrations are given by the solid black and gray curves, respectively. The velocity is given by the dashed curve.

in Fig. 5(b). The presence of flow qualitatively alters the dispersion relation [Fig. 5(c)]. For given values of $D_{a} / D_{s}$, $\rho_{s}$, and $\rho_{a}$, increasing Pe broadens the band of wave numbers for which $\operatorname{Re}[\lambda(k)]>0$. The steady concentration profile features peaks in activator concentration that contract fluid into them, providing an influx of activator, as shown in Fig. 5(d). This stabilizes the peaks against diffusion in a manner similar to the previous example, even in regimes where the activator diffuses faster than the inhibitor. Thus, active stress-driven flow serves to broaden the pattern-forming region of parameter space, giving patterns not only in concentration of chemical species but also in mechanical stress and fluid motion.

We have developed a general hydrodynamic theory of chemically regulated active stress in viscous fluids and identified general principles of pattern formation in such systems. This approach can be extended to higher dimensions and to include polar and nematic order, viscoelasticity, and other reaction systems. In the two scenarios presented here, peaks in concentration of stress activator are amplified by advective influx due to active flows. This simultaneously depletes activator from areas around a concentration maximum. Thus, active stress-driven flow provides both the local activation and lateral inhibition required to form stable patterns $[11,12]$. This mechanism leads to pattern formation even in the absence of chemical reactions. Furthermore, in classical reaction-diffusion systems, coupling to active stress alters the parameter space in which patterns occur. These mechanisms are of particular interest for biological pattern formation in which material flow occurs. Many developmental processes in the cell and tissue scale involve both patterning and flows. Examples include cellular polarization, gastrulation, and cell sorting $[2,7]$. We expect that integration of active hydrodynamics with molecular signaling processes will be an important theme for future studies of such biological processes.

We thank A. E. Carlsson, M. Depken, P. W. Dondl, N. W. Goehring, P. Khuc Trong, E. M. Nicola, J. M. R. Parrondo, and G. Salbreux for useful discussions. J. S. B. acknowledges the Human Frontier Science Program for funding.

[1] A. M. Turing, Phil. Trans. R. Soc. B 237, 37 (1952).

[2] T. Mammoto and D. E. Ingber, Development (Cambridge, U.K.) 137, 1407 (2010).

[3] K. Kruse et al., Phys. Rev. Lett. 92, 078101 (2004); Eur. Phys. J. E 16, 5 (2005).

[4] R. A. Simha and S. Ramaswamy, Phys. Rev. Lett. 89, 058101 (2002).

[5] A. Ahmadi, M. C. Marchetti, and T. B. Liverpool, Phys. Rev. E 74, 061913 (2006).

[6] G. Salbreux, J. Prost, and J.-F. Joanny, Phys. Rev. Lett. 103, 058102 (2009).

[7] M. Mayer et al., Nature (London) 467, 617 (2010).

[8] In the biological context of the actomyosin cell cortex, this simple choice corresponds to a membrane-bound regulator of active stress that does not exchange with the cytoplasm.

[9] L. D. Landau and E. M. Lifshitz, Mechanics (ButterworthHeinemann, Burlington, MA, 1982), 3rd ed.

[10] L. M. Pismen, Patterns and Interfaces in Dissipative Dynamics (Springer-Verlag, Berlin, 2006).

[11] A. Gierer and H. Meinhardt, Kybernetik 12, 30 (1972).

[12] A. J. Koch and H. Meinhardt, Rev. Mod. Phys. 66, 1481 (1994). 